\title{
The Emergency Medical System in Albania.
}

The Mission of the National Emergency Medical Center (NEMC)

Skënder Brataj ${ }^{1}$, Agron Dogjani²

\begin{abstract}
Territorial Emergency Service is part of NEMS. It belongs to the only telephone number 127 (112) and it is free of charge. It represents the guarantee of medical emergency service provided by law 147/2014 (For Emergency Medical Service) and with the Council of Ministers Decision (CMD) No.933 date 29.12.2014 on Approval of the Organizational Structure of the National Center of Medical Emergency, CMD. Nr. 250, date 30.3.2016 and aims to guarantee the response to emergencies and medical emergencies, from the moment of receiving the call until their resolution, throughout the territory of the Republic of Albania. This paper aims to give an introduction and to analyse the emergency medical system's mission in Albania.

Keywords: National Emergency Medical Center, NEMC, mission
\end{abstract}

*Corresponding Author: Agron Dogjani

$\equiv \triangle$ E-mail: agrondogjani@yahoo.com

${ }^{1}$ The National Emergency Medical Center, Tirana, Albania

2University Hospital of Trauma, Tirana, Albania 


\section{Full Text}

Territorial Emergency Service is part of NEMS. It belongs to the only telephone number 127 (112) and it is free of charge.

-It represents the guarantee of medical emergency service provided by law 147/2014 (For Emergency Medical Service) and with the Council of Ministers Decision (CMD) No.933 date 29.12.2014 on Approval of the Organizational Structure of the National Center of Medical Emergency, CMD. Nr. 250, date 30.3.2016 and aims to guarantee the response to emergencies and medical emergencies, from the moment of receiving the call until their resolution, throughout the territory of the Republic of Albania.

-Decreases the timing of therapy from the moment of symptoms to their resolution, in order to reduce the avoidable deaths, reducing disability, as traumatic and nontraumatic.

-Prevents the immediate clinical evolution of the disease and significantly reduces their return to normality.

-Coordinates immediate centralization of acute and critical patients from territory to appropriate hospitals without wasting time, reducing the percentage of mortality and disability.

- It is built in the entire territory according to national standard parameters in accordance with international standards. Makes the coordination between the cities and villages based on the needs that may arise, such as floods, earthquakes, and mass erosion of various natures.

- It is a structure built by: the operating rooms equipped with the appropriate technology for obtaining and evaluating the demand for assistance, sending the vehicle with the proper crew and stations in a dispersed territory in order to shorten as far as possible the time of arrival to the patients with the personel concerned.

- It is a structure with its public organogram, incorporated under the law, specialized and formed to be able to respond to any type of medical emergencies. Its structure includes doctors, nurses, support staff, technical and administrative staff, suitable for 24-hour service as per operating room as well as for territorial stations.

- It is a structure equipped with complex tools and equipment such as technological, informatics and others, which must be homogeneous across the territory because they guarantee the responsibility for direct management of all of the above resources while guaranteeing the right quality at critical service moments.

- It's composed of ground vehicles, flight and water vehicles (sea, lake, rivers), such as ambulances, helicopters, boats or speedboats to respond to medical emergencies.

- Provides assistance in case of festive sports events or at times of seasonal and tourist overcrowding, by coordinating them or by overseeing other organizations that may be there to provide assistance. -It makes protocols with other health and nonhealth facilities, such as police, firefighters, civil defense and others, which may be involved with emergencies in general.

- Conducts continuous activity of its own personnel according to clinical medical records in accordance with international and contemporary literature.

- Promotes and provides throughout the national territory uniform standards of 
education, training, practice, first aid, cardio-pulmonary resuscitation, immediate defibrillation.

-These programs address the population starting from school age, thus guaranteeing its active participation in the defibrillation program.

-It is an integral part of Emergency Medicine at the University of Medicine as well as Emergency and Anesthesia-Resuscitation specialty trainings. It also organizes and participates in Masters, scientific researches in the field of EmergencyTerritorial, Emergency Medicine and Hospital Emergencies.

- Operates under the most modern systems which control and verify the quality, in order to orient towards continuous improvement of clinical management, thus reducing the risk of medical errors especially in acute patients.

- Promotes and participates actively with other national and international associations in the design and implementation of pathology networks (cardiac arrest, acute coronary syndrome, severe trauma, acute respiratory failure, stroke, other epidemiological surveillance)

\section{Analysis of the Emergency Medical System in Albania.}

The birth of a new service or reorganization begins with an analysis of its current state. So before we begin to show how this service works today, we will begin to explain how it was before the start of the work of the Coordination Unit at the National Emergency Medical Center. A service like that of the "Emergency Medicine" is irreplaceable and must be infallible. The key indicators highlighted the problems of this service, which we are explaining according to the reality presented in 2014. At disposal we had the material prepared by the working group run by USAID and the working group of the NEMC, which provided the data and a more detailed analysis of the final conclusions.

Below is the material, the current state of service, international standards and the recommendations of the working group.

\section{USAID:}

\section{Pre-Hospital Care}

Pre-hospital care is a vital element of trauma systems, what happens at this level often has a direct impact both in initial treatment and in subsequent outcomes. The trauma care system's preprimary elements should provide easy access and immediate response from qualified professionals responsible for assessing, stabilizing, selecting and transporting to the nearest foster care institution.

\section{Description of communication standards:}

The communication systems provide essential coordination between the components of NEMC and the trauma care system. An efficient communication plan provides national coverage and coordination of NEMC communications. Areas with problems in signal's interference and uncovered areas should be minimized. The communication component of the 
trauma care system should focus on access to the system, dispatch of NEMC dispatch assistance to persons who are not at the scene, the overall communication links of the NEMC and the activities of the program for improving the quality. Immediate identification of an accident or an event that has caused damage to people, requires the planning and implementation of appropriate methods such as accident and damage surveillance technology (eg presence of vehicle transmitters) and public awareness programs on access to the NEMC system.

Easy access to the system, through a single coordinated system such as 9-1-1 system, or a reinforced telephone system 9-1-1 that enables automatic caller location, should be available in all locations. The distribution of pre-hospital staff should be organized in every region, in order to facilitate coordination of emergency response throughout the system. Dispatchers must have completed a medical emergency dispatch course by being prepared for the most appropriate response to the emergency medicine service (EMS). The Dispatch Center should provide call filtering and structured questions to ensure the optimum use of personnel, equipment, and spotting. The needs of the injured person must match the existing resources of the EMS. In addition, all callers should be provided with pre-arrival instructions, according to some protocols. Special protocols should be developed for responding to one or more patients as well as for accidents with multiple victims. The NEMC distribution system should be run by a physician and incorporate regular collegial medical reviews as well as ongoing quality improvement. If conditions permit, the system must incorporate the best available technology, including computer aided dispatch. The trauma care system plan should incorporate the possibility of communication between pre-hospital care providers, medical practitioners or medical directors and staff of the trauma care provider institution.

The necessary interconnections include communication between ambulances, ambulance-hospital and hospital-hospital, as well as ease of communication with the police and other agencies at the scene. Currently, we have available technology and equipment to provide effective online communications in most areas.

\section{Summary of ascertained findings during the assessment for the period in question.}

Currently, the pre-hospital system is the most necessary system, so that the trauma system for Albania can be successful. There are many ways of announcing ambulances in the event of injury. There are areas where a national telephone number is used, 112, to ask for help or to notify the police. However, this number is not always used and there are other local or regional numbers that everyone needs to know for access to emergency services. For example, 112 is not operational in Durres, as people call to mock and communicate false alarms, abusing the system; this made the system shut down and another number implemented. After a week this second number was resealed. Most respondents stated that system 112 is a realistic option and needs to be improved so that wherever you are, can call this number and after registering your 
localization, the call is directed to a communication center of the region, from where the closest ambulance can be sent. In the opinion of all respondents, GPS and communication systems need to be improved. Given that there are no formal communications or distribution centers, the training for staff dealing with the reception of these calls is very limited or almost nonexistent.

\section{NEMC}

Tirana had three phones available to its citizens. These phones were not always in service and were not enough for a city like Tirana; three phones for about a million people. Thus, the possibility of controlling the need for medical help was impossible. In the rest of the country 168 phone numbers were not active. The answer to the citizens was under the responsibility of a person, and the most common response was to go to the hospital. The number of ambulances visiting homes was only one or two for each city, while it was not present in any village and was partially covered by cities with one-hour intervention and partial coverage of this service. The city of Tirana had only three ambulances in the field for 24 hours. There was a lack of equipments for performing cardiopulmonary resuscitation. Trainings were sporadic and very limited for the medical emergency sector. Lack of indicators that control the quality of medical performance was a deficiency that did not allow data collection and analysis.

The coordination center served only one room with telephones, but not a center coordinating all the assets in a single national system.

The documentation was almost nonexistent and there was no territorial clinical cartel, but simply a recipe where the surname was written and a diagnostic hypothesis.

The ambulance control system was missing and there was no coordination between them. Lack of uniform did not identify a unified service.

\section{Recommendations: USAID}

It is recommended to use the existing adult infrastructure and to develop a sophisticated communication network as well as the use of 112 in the whole country. In case of misuse of this number or false alarms, strict penalties must be applied. GPS and other communication technologies can be used to send ambulances that are closer to the location of the accident. The time factor is very important in the trauma process and patients need to be traced and transported rapidly to the trauma center by professionals. A national call center, equipped with a dispatcher, should be established to have links with regional centers to disperse the police and ambulances to the scene of the accident at a rapid pace. Technology and cellular systems can help support the communications network for distribution of ambulances. It is recommended that staff at dispatch centers of communication, should operate on standard protocols by distributing and making callers the right questions. There are some standardized training programs that can be used. Standards sets with 
protocol cards of dispatch centers are available. Below we are listed some electronic addresses with examples of protocols and training information for the staff of the dispatch center.

http://www.911dispatch.com/info/emd/emdprs.html

http://www.local4260.com/home/911sys.html http://www.prioritydispatch.net//

The Teletrauma Network can also be used as an opportunity to carry out the training of the staff of the call center. The curriculum can be loaded and embedded in a center and then distributed to other regional dispatch centers.

Recommendations: NEMC
Extending the unique number 127 throughout the country after the creation of the Coordination Unit is the only way to operate this service. Completion of all medical teams on the ground with a medical emergency phone number solves the problem of instant communication. Setting up a radio communication system takes place at a second moment.

Filling the ambulance fleet with the GPS system would create the possibility of locating and controlling them by providing real-time service coverage. Starting the training of medical and support personnel is the only way to guarantee life and quality of life for the welfare of this service.

Establishing quality control indicators is a necessity for improving medical emergency service.

This is the introductory material prepared for the Medical Emergency Service. The current status of this system follows in the next issue. 